\title{
Respuesta cutánea de lactantes a 2 y 10 unidades de tuberculina
}

\author{
M.C. Miguel O'Ryan G. ${ }^{2}$; M.C. Teresa Gómez P.' ; M.C. Juan Tapia V.' ; \\ M.C. Marcos Sáez G, ${ }^{1}$; M.C. Eduardo Talesnik G.';
}

E.L. Santiago Rivero D. ${ }^{2}$; E.U. Sandra Vilches J. ${ }^{3}$; E.U. Verónica Capdeville M. ${ }^{3}$

\section{Tuberculin response to $2 \mathrm{TU}$ and $10 \mathrm{TU}$ doses in infants}

\begin{abstract}
A study was done in 322 healthy, well nourished infants, 3 to 18 months old from day care centers of metropolitan Santiago, Chile, that were given BCG immunization in their neonatal period: $304(94,4 \%)$ of them show. ed BCG scars and were included in a double blind open study, to determine the cutaneous responses to 2 TU and 10 TU tuberculin (PPD). There were no differences in the mean size of cutaneous reactions nor in percent positive responses ( $>10 \mathrm{~mm}$ ), but cutaneous reactions $>15 \mathrm{~mm}$ were more frequent in infants tested with PPD 10 TU. In 184 out of 304 infants $(60.5 \%$ ) tuberculin reactions were negative ( $10 \mathrm{~mm}$ ). Eighteen out of 322 infants $(5.6 \%)$ that didn't show BCG scars were injected with PPD 2 TU: tuberculin reactions sized 6 to $9 \mathrm{~mm}$ were recorded in $3 / 18$ of these infants but none of them attained $10 \mathrm{~mm}$. Fifty five infants whose tuberculin reactions were $5 \mathrm{~mm}$ or less were retested: a booster effect was likely from the appearence of enhanced dermal response after a second tuberculin test done with the same or higher tuberculin strength than the first one in $12 / 47$ of these infants with BCG scar $(25 \%)$ and in only $1 / 8$ such subjects without BCG scar.
\end{abstract}

(Key words: BCG vaceination, tuberculin response.)

1. Departamento Pediatría, Escuela de Medicina, Pontificia Universidad Católica de Chile.

2. Departamento de Inmunología Clínica y Reumato. lógica, Escuela de Medicina, Pontificia Universidad Católica de Chile.
3. Enfermeras universitarlas. Servicio de Pedigtría, Hospital Clínico Universidad Católica de Chile.

Trabajo financiado por la Comisión Investigación Científica Facultad de Medicina, Pontificia Universidad Catálica de Chile. Concurso de Investigación para Médicos Becarios. 
La inmunización con BCG induce sensibiliza. ción a tuberculina (PPD), la cual puede variar tanto con el tipo de vacuna y técnica de administración como por factores del huésped ${ }^{1-3}$. Esta prueba cutánea de hipersensibilidad retardada continúa siendo de amplio uso en estudios epidemiológicos y como ayuda en el diagnóstico de tuberculosis ${ }^{4}$.

Publicaciones previas han demostrado diferencias en la reacción cutánea a la vacuna BCG $y$ en la respuesta a tuberculina en niños vacunados en el período de recién nacido ${ }^{5-7}$. Se diseñó un estudio en una población de lactantes de 3 a 18 meses, sanos, eutróficos provenientes de salas-cunas de la Región Metropolitana, con antecedentes de haber recibido, en el período neonatal, vacuna BCG liofilizada, actualmente en uso $^{8}$, para determinar el porcentaje de niños con respuesta cutánea $\mathrm{BCG}$ positiva (cicatriz) y efectuar en éstos un estudio doble ciego, aleatorio, con el objeto de investigar eventuales variaciones en la reacción cutánea a dos distintas concentraciones de tuberculina (2 UT y 10 UT) y, en los lactantes vacunados pero sin cicatriz cutánea $\mathrm{BCG}$, determinar la respuesta a PPD 2 UT. Asimismo, se intentó determinar si se producen incrementos en las respuestas cutáneas a una nueva dosis de tuberculina en lactantes cuya respuesta inicial fuese negativa.

\section{MATERIAL Y METODOS}

Durante el período comprendido entre julio de 1986 y agosto de 1987 se visitaron 32 salas-cunas de la Región Metropolitana. Se obtuvo el consentimiento de los padres para ingresar los lactantes al estudio. Se catalogó el estado nutricional según tablas del Centro Nacional de Estadísticas en Salud de E.U.A. Se excluyeron los lactantes con relación peso/talla y/o peso/ edad bajo el percentil $10^{\circ}$ y los que estuviesen cursando enfermedades crónicas, infecciones recurrentes, usando fármacos o tuviesen antecedentes de contacto familiar con enfermos tuberculosos. De esta manera se identificaron 322 lactantes eutróficos entre 3 y 18 meses con antecedentes de haber sido vacunados con BCG en el período neonatal, de los cuales 304 tenían cicatriz BCG y 18 carecían de ella.

El PPD 2 UT y 10 UT fue preparado y suministrado por el Instituto de Salud Pública de Chile. Los lactantes con cicatriz cutánea BCG fueron inoculados en forma aleatoria con PPD 2 UT o 10 UT por una enfermera universitaria entrenada en la técnica, utilizando jeringa de tuberculina. La lectura fue realizada $48 \mathrm{~h}$ después, midiendo el diámetro transverso de la induración, por otro de los autores, el cual no conocja la concentración de PPD utilizada. Los 18 lactantes sin cicatriz cutánea BCG recibjeron exclusivamente PPD 2 UT en condicio- nes semejantes a las recién descritas. Se consideró respuesta cutánea positiva una induración palpable de diámetro $>10 \mathrm{~mm}^{4}$.

A 55 lactantes con respuesta cutánea $65 \mathrm{~mm}$ en una primera prueba de PPD 2 UT o 10 UT se les administró cuatro semanas después un segundo PPD: a 23 de ellos, con cicatriz cutánea BCG positiva, en mayor concentración que en la primera prueba; a 24 también con brote cutáneo BCG, en igual o menor concentzación que el injicial y a 8 niños, sin cicatriz cutánea BCG, una segunda dosis de PPD 10 UT, habiendo sido, como se djjo, inoculados originalmente con PPD 2 UT.

Se consideró conversión si se registraba un incremento, en el tamaño de la reacción, de al menos $6 \mathrm{~mm}$ desde $<10 \mathrm{~mm}$ a $>10 \mathrm{~mm}$. Se consideró que no habían cambios en la respuesta si ocurtían variaciones de $\leqslant 2 \mathrm{~mm}$ entre el primero y el segundo PPD ${ }^{10,1}$.

En el análisis esiadístico se utilizó la prueba de Stu. dent para muestras pareadas y el ensayo no paraméttico de Friedman para muestras de tamaño reducido ${ }^{13}$.

\section{RESULTADOS}

Ingresaron al estudio 322 lactantes cuya edad promedio era 12 meses, eutróficos, con relación peso/talla promedio en el percentil $\$ 2$.

Se identificaron 304 lactantes con cicatriz cutánea BCG, 135 de los cuales fueron inoculados con PPD 2 UT y 169 con PPD 10 UT. No hubo diferencias en cuanto a edad, sexo y estado nutricional entre ambos grupos, tampoco al compararlos según el promedio del tamaño de la respuesta cutánea $(6,93 \pm 5,42 \mathrm{~mm}$ para los que recibieron PPD 2 UT vs 7,22 $\pm 6,11 \mathrm{~mm}$ con PPD 10 UT), nj en los porcentajes de respuestas cutáneas positivas $(\geqslant 10 \mathrm{~mm}$ ): $38,5 \%$ para $P P D$ 2 UT vs 40,2\% para PPD I0 UT. Sólo se encontraron diferencias significativas para las reacciones $\geqslant 15 \mathrm{~mm}$ que fueron más frecuentes en el grupo al cual se le administró PPD I0 UT (tabla 1). En la figura 1 observa el histograma de distribución para respuestas cutáneas a PPD 2 UT y 10 UT.

En tabla 2 se muestran el porcentaje de respuestas cutáneas positivas y la media del tamaño de la reacción cutánea según djstribución etaria. No se observan diferencias estadística. mente significativas al comparar por distintos grupos de edades lactantes inoculados con PPD 2 UT y 10 UT.

En total, 60,5\% de los niffos con antecedentes de vacuna BCG y cicatriz tuvieron reacciones PPD 2 UT o 10 UT negativas $(<10 \mathrm{~mm})$.

Se aplicó un análisis de varianza y comparaciones múltiples, por separado para los grupos 
Tabla 1

Tamaño de respuesta cutánea a PPD 2 UT y 10 UT en 304 lactantes con cicatriz BCG

\begin{tabular}{cccccc}
\hline $\begin{array}{c}\text { Tamaño de respuesta } \\
\text { (mm) }\end{array}$ & $\begin{array}{c}\text { PPD } \\
\mathbf{n}\end{array}$ & $\begin{array}{c}2 \text { UT } \\
\%\end{array}$ & $\begin{array}{c}\text { PPD } \\
\mathbf{n}\end{array}$ & $\begin{array}{c}10 \text { UT } \\
\%\end{array}$ & $\mathrm{p}^{*}$ \\
\hline $0-5$ & 49 & 36,3 & 70 & 41,4 & NS \\
$6-9$ & 34 & 25,2 & 31 & 18,4 & NS \\
$10-14$ & 41 & 30,4 & 40 & 23,6 & NS \\
$>-15$ & 11 & 8,1 & 28 & 16,6 & $<0,05$ \\
\hline
\end{tabular}

$\mathrm{p}^{*}=$ Prueba $\mathrm{t}$ de Student.

PPD 2UT

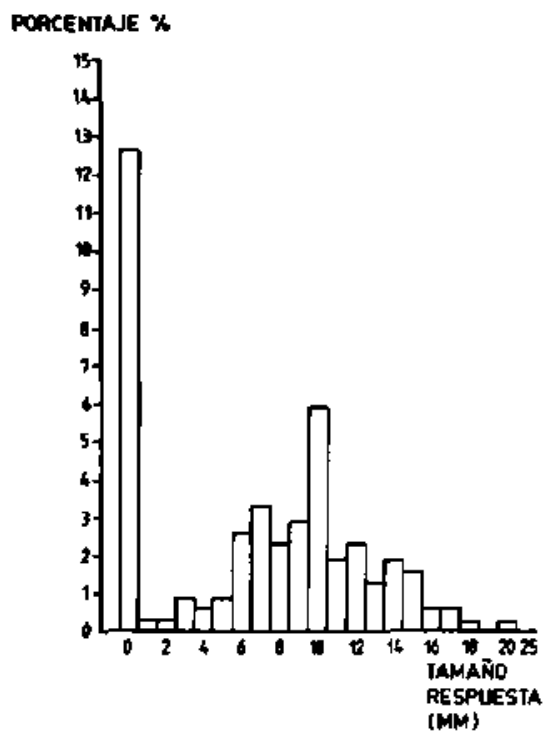

PPD 10UT

\section{PORCENTAJE \%}

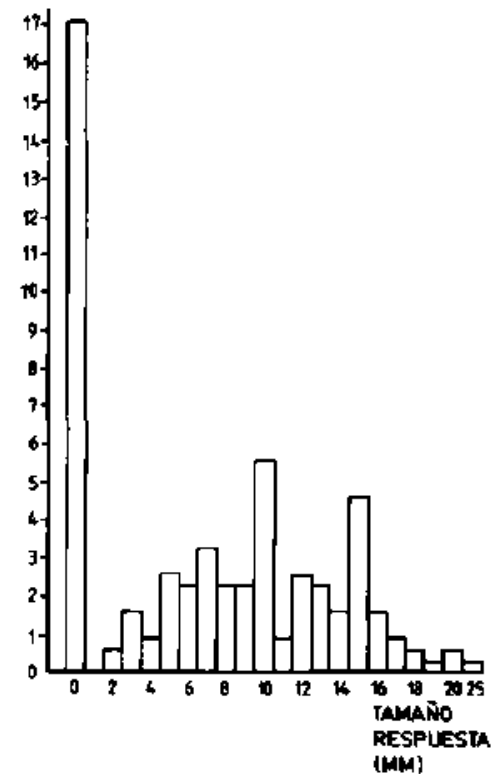

Fignar 1: Histograma de distribución de respuestas cutáneas a PPD 2 UT y 10 LT en 304 lactantes con cicatriz cutánea BCG de la Región Metropolitana, julio 1986-agosto 1987.

Tabla 2

Tamaño respuesta cutánea a PPD 2 UT y 10 UT según distribución etaria en 304 lactantes con cicatriz BCG

\begin{tabular}{|c|c|c|c|c|c|c|c|}
\hline $\begin{array}{c}\text { Edad } \\
\text { (meses) }\end{array}$ & $n$ & $\begin{array}{c}\bar{x} \pm D E \\
(\mathrm{~mm})\end{array}$ & $\begin{array}{l}\text { Respuesta cutá- } \\
\text { nea positiva } \\
(>10 \mathrm{~mm}) \%\end{array}$ & $\mathbf{n}$ & $\begin{array}{c}\bar{x} \pm \mathrm{DE} \\
(\mathrm{mm})\end{array}$ & $\begin{array}{c}\text { Respuesta cutá- } \\
\text { nea positjva } \\
(\geqslant 10 \mathrm{~mm}) \%\end{array}$ & $\mathrm{p}^{*}$ \\
\hline $3-6$ & 19 & $10,37 \pm 3,82$ & 63,1 & 16 & $9,00 \pm 5,98$ & 50,00 & NS \\
\hline $7-9$ & 21 & $6,33 \pm 5,30$ & 38,1 & 19 & $9,10 \pm 5,66$ & 57,9 & NS \\
\hline $10-12$ & 28 & $6,86 \pm 5,54$ & 39,3 & 30 & $6,70 \pm 5,84$ & 33,3 & NS \\
\hline $13-15$ & 37 & $5,83 \pm 5,82$ & $29, ?$ & 37 & $7,19 \pm 5,84$ & 40,5 & NS \\
\hline $16-18$ & 30 & $6,56 \pm 5,21$ & 33,3 & 67 & $6,50 \pm 6,49$ & 35,8 & IS \\
\hline
\end{tabular}


inoculados con PPD 2 UT y 10 UT (prueba de Student-Neuman-Keuls) y se demostró una djferencia estadísticamente significativa sólo para los inoculados con PPD 2 UT entre el grupo de 3 a 6 meses $y$ los otros grupos etarios inoculados con la misma concentración de tuberculina. No hubo diferencias entre los distintos grupos según distribución por edad en niffos a los que se les administró PPD 10 UT.

No se encontró brote cutáneo de $\mathrm{BCG}$ en 18 de $\operatorname{los} 322$ niños $(5,6 \%)$. No se establecieron diferencias en cuanto a edad, sexo y estado nu. tricional en relación al grupo con brote cutáneo BCG. El grupo completo que carecía de cicatriz cutânea $\mathrm{BCG}$ fue inoculado con PPD 2 UT: $16,3 \%(3 / 18)$ tuvieron respuestas cutáneas entre 6 y $9 \mathrm{~mm}$ y $83,4 \%(15 / 18)$ entre 0 y $5 \mathrm{~mm}$ de diámetro.

Al comparar el promedio del tamaño de la reacción cutánea entre los niños inoculados con PPD 2 UT, con cicatriz cutánea positiva $(n=135)$ y $\sin$ ella $(n=18)$, se estableció una diferencia estadisticamente significativa (PPD 2 UT con cicatriz $\mathrm{BCG}=6,92 \pm 5,42 \mathrm{~mm}$ vs. PPD 2 UT sin cicatriz BCG $=2,94 \pm 2,90$ mm $p<0,001$ ), lo que fue corroborado con prueba no paramétrica de Wilcoxon.

Los resultados de las reacciones cutáneas a dos inoculaciones de tuberculina, con un mes de intervalo entre ellas, cuando la respuesta era $\leqslant 5 \mathrm{~mm}$ a la primera, se resumen en la tabla 3 . Ninguno de los nifnos presentó disminución de la reactividad al repetir la dosis de tuberculina.
Se demostraron diferencias estadísticamente significativas ante segundas inoculaciones de PPD de mayor o igual concentración que la inicial en lactantes con cicatriz cutánea BCG positiva.

Presentaron canversión positiva $12 / 47(25 \%)$ del total de lactantes con cicatriz BCG positiva inoculados con un segundo PPD, en tanto que esto ocurrió en $1 / 8(12,5 \%)$ de aquellos con cicatriz cutánea negativa (tabla 3 ).

\section{DISCUSION}

No se establecieron diferencias en las respues. tas cutáneas al administrar PPD 2 UT o 10 UT en lactantes vacunados con BCG liofilizada con cicatriz cutánea presente. $\mathrm{El}$ haber encontrado un mayor número de reacciones cutáneas $\geqslant 15$ mm entre los niños inoculados con PPD 10 UT sugiere que al existir memoria inmunológica la mayor concentración del antígeno influye sólo para las respuestas de mayor tamaño. La población estudiada parece adecuada, pues eran lactantes menores de 18 meses, eutróficos, en los que se ha demostrado la más alta proporción de reacciones positivas al inóculo de $\mathrm{PPD}^{6,7}$, todos con el antecedente de haber recibido vacuna BCG en el período de recién nacido, con cicatriz cutánea inducida por esta vacuna. Se excluyeron aquellos lactantes que pudiesen tener factores individuales capaces de alterar su reactividad a la tuberculina ${ }^{2}$.

Tabla 3

Respuesta cutánea al isocular segundo PPD en 55 lactantes vacunados con $B C G$ al nacer, con respuesta $\varangle 5 \mathrm{~mm}$ a primer PPD

\begin{tabular}{|c|c|c|c|c|c|c|c|c|}
\hline & Primer PPI & (PPD) & & Segundo & $D$ (PPD2) & PPD 1-PPD2 & & \\
\hline $\begin{array}{l}\text { Cica- } \\
\text { triz } \\
\text { BCG }\end{array}$ & $\begin{array}{l}\text { Concen- } \\
\text { tración }\end{array}$ & $\mathbf{n}$ & $\begin{array}{c}\bar{x} \\
(\mathrm{~mm})\end{array}$ & $\begin{array}{l}\text { Concen- } \\
\text { tración }\end{array}$ & $\begin{array}{c}\overrightarrow{\mathrm{x}} \\
(\mathrm{mm})\end{array}$ & $\mathrm{p}^{*}$ & $\begin{array}{l}\text { Conversión } \\
\text { positiva } \\
(\%)\end{array}$ & $\begin{array}{l}\text { Ausencia } \\
\text { de cambio } \\
(\%)\end{array}$ \\
\hline Positiva & $2 \mathrm{UT}$ & 23 & $0,56 \pm 1,37$ & $10 \mathrm{UT}$ & $6,17 \pm 5,14$ & $<0,0001^{*}$ & $7(30,4)$ & $13(56,5)$ \\
\hline Positiva & $2 \mathrm{UT}$ & $B$ & $1,25 \pm 2,16$ & $2 \mathrm{UT}$ & $3,87 \pm 3,63$ & $<0,05$ & $1(12,5)$ & $5(62,5)$ \\
\hline Positiva & $10 \mathrm{UT}$ & 8 & $0,37 \pm 0,99$ & $10 \mathrm{UT}$ & $4,37 \pm 4,81$ & $<0,05 * *$ & $2(25)$ & $4(50)$ \\
\hline Positiva & $10 \mathrm{UT}$ & 8 & $1,25 \pm 2,16$ & $2 \mathrm{UT}$ & $3,62 \pm 5,42$ & NS ** & $2(25)$ & $6(75)$ \\
\hline Negativa & $2 \mathrm{UT}$ & 8 & $0,40 \pm 1,10$ & $10 \mathrm{UT}$ & $3,50 \pm 4,60$ & NS ** & $1(12,5)$ & $S(62,5)$ \\
\hline
\end{tabular}

$p^{*}=$ Prueba $\mathbf{t}$ de Student.

** = Test no paramétrico de Friedman. 
Es notable que $60,5 \%$ del total de lactantes estudiados tengan respuesta negativa al ser inoculados con PPD 2 UT y 10 UT. Estos resul. tados son distintos a los obtenidos por estudios anteriores, pues existen comunicaciones con $76 \%$ de respuestas $\geqslant 10 \mathrm{~mm}$ en lactantes de 3 meses $^{13}$ y $79 \%$ de respuestas $\geqslant 5 \mathrm{~mm}$ a $\operatorname{los} 6$ meses de edad? El premedio del tamaño de la reacción obtenida en nuestro trabajo es semejante al descrito por Dotti ${ }^{5}$, utilizando también vacuna BCG liofilizada. Por otro lado, en dos estudios nacionales, usando este mismo tipo de vacuna, se comunicaron tanto menores porcentajes de respuestas $\geqslant 10 \mathrm{~mm}$ cono promedios de tamaño de la reacción cutánea, en lactantes de 4 meses del Area Metropolitana, con 0 sin cicatriz cutánea. Factores nutricionales y genéticos ${ }^{16}$, el tipo de vacuna utilizada ${ }^{14} \mathrm{y}$ aun interferencia por anticuerpos maternos a micobacterias atípicas ${ }^{4}$ se han invocado para explicar estas diferencias. En nuestro estudio la población de niños era eutrófica y la vacuna suministrada en el periodo neonatal fue la forma liofilizada actualmente en $\mathrm{uso}^{8}$, para la cual se ha demostrado que existen adecuados controles de calidad ${ }^{14}$ y ventajas sobre la vacuna líquida, en cuanto a estabilidad ${ }^{6}$; sin embargo, induce reacciones cutáneas a la tuberculina de menor tamaño que la forma líquida ${ }^{14}$.

La disminución en la respuesta cutánea a PPD en niños vacunados con BCG al nacer, mayores de 6 meses, ha sido descrita previamen$t^{7}$. La confimmamos sólo al utilizar PPD 2 UT, pero no con PPD 10 UT, diferencias cuya interpretación no conocemos.

La ausencia de brote cutáneo a la vacuna fue encontrada en $5,6 \%$ de los niños estudiados, en contraste con $24,8 \%$, como se describió en niños de origen asiático ${ }^{16}$, lo que fue atribuido a menor peso de nacimiento, edad gestacional y nivel socioeconómico. Por otro lado, demostramos una clara diferencia en la respuesta cutánea a PPD 2 UT entre lactantes con y $\sin$ cicatriz cutánea BCG. La respuesta inmunitaria a esta vacuna en lactantes vacunados sin la cicatriz no se ha caracterizado completamente. En nuestro estudio muy pocos lactantes de este tipo mostraron respuestas cutáneas de 6 a $9 \mathrm{~mm}$ al primer PPD 2 UT. Al inocular la segunda dosis de PPD con 10 UT en aquellos cuya respuesta era $\leqslant 5 \mathrm{~mm}$ al primer PPD 2 UT no hubo aumento significativo en el tamaño de la reacción y sólo $1 / 8$ tuvo conversión positiva (tabla 3 ), en tanto que en lactantes de origen asiático inmunizados con BCG y ausencia de brote cutáneo a la vacuna, se comunicó $40 \%$ de respuestas cutáneas $\geqslant 5 \mathrm{~mm}$ a PPD $10 \mathrm{UT}^{16}$.

El incremento en la respuesta cutánea ante dosis sucesivas de tuberculina en niffos vacunados con BCG se ha atribuido a un estímulo antigénico inicial dado por la vacuna, reforzado por la primera tuberculina, lo cual produciria respuestas de mayor intensidad con dosis sucesivas, fenómeno descrito en escolares daneses ${ }^{17} \mathrm{y}$ chilenos $^{18}$. En lactantes con respuesta inicjal a tuberculina francamente negativa ( $\leqslant 5 \mathrm{~mm}$ ), tanto la presencia de cicatriz cutănea BCG como el empleo de una segunda dosis de tuberculina de mayor o igual concentración que la inicial constituyeron probablemente las condiciones para obtener el aumento observado en esta respuesta de hipersensibilidad retardada.

Sería importante desarrollar investigaciones que permitiesen disponer en el futuro de pruebas específicas para diferenciar las infecciones causadas por Mycobacterium tuberculosis y por $\mathrm{BCG}$, como también técnicas de laboratorio para medir en forma más precisa la respuestas inmune a la vacuna $B C G$ en individuos sin cicatriz cutánea inducida por ésta.

\section{RESUMEN}

Se estudiaron 322 lactantes sanos, eutrófi$\cos$, entre 3 y 18 meses de edad, que habian sido inmunizados con $\mathrm{BCG}$ en el período neonatal y procedian de salas-cunas de la Región Metropolitana. Entre ellos, $304(92,4 \%)$ tenian cicatriz BCG y fueron ingresados a un estudio doble ciego aleatorio, para determinar eventuales va. riaciones en las respuestas cutáneas a PPD 2 UT y 10 UT. No se encontraron diferencias en el tamaño de las respuestas cutäneas ni el porcentaje de respuestas positivas $(10 \mathrm{~mm})$. excepto para las reacciones $\geqslant 15 \mathrm{~mm}$, que fueron más frecuentes en los nifros inoculados con PPD 10 UT. En $184 / 304(60,5 \%)$ se obtuvo respuesta cutánea negativa $(\leqslant 10 \mathrm{~mm})$. Los $18 / 322(5,6 \%)$ que no tenían cicatriz BCG fueron inoculados con PPD 2 UT: ninguno mostró respuesta cutánea positiva. y en $3 / 18$ ésta fue de 6 a $9 \mathrm{~mm}$. Al administrar una segunda dosis de PPD a 55 lactantes cuya respuesta inicial había sido $\leqslant 5 \mathrm{~mm}$, se obtuvieron reacciones de mayor tarnaño que en la primera en los 
niños que tenían cicatriz BCG cuando la segunda dosis era de igual o mayor potencia que la primera, sugiriendo un mecanismo de refuerzo.

(Palabras clave: vacuna BCG, reacción tuberculínjea.)

\section{REFERENCIAS}

1. Snider D.E.: Bacille Calmette - Guerin Vaccinations and Tuberculin Skin Tests. JAMA 1985; 23: 3438-3439.

2. Starke J: Modern Approach to the Diagnosis and Treatment of Tuberculosis in Children. Pediat Clin North Am 1988; 35; 441-464.

3. Chaparas S.D., Vandiviere H.M., Melvin I., Koch H. Becker C.: Tuberculin Test Variability with the Mantoux Procedure. Am Rev Resp Dis 1985: 132: $175-177$.

4. Farga $V_{::}$Tuberculosis. Santiago, Editorial Mediterráneo. $1989 ; 66-69$.

5. Dotti De., Amengual A.: Evaluación de los resultados de una prueba de campo con vacuna BCG liofilizada en lactantes. Rev Arg Tuberculosis, Enfermedades Pulmonares y Salud Pública 1982; 43: $23 \cdot 30$.

6. Miller FJ. w.: Tuberculosis in Children. Churchill Livingstone Edinburgh, England, 1982;55-65.

7. Willshaw M.E., Santiagess R., Infante R., Vargas M.- Duración de la Alergia Inducida por el BCG. Rev Chil Pediatr 1966; 37: 174-179.

8. Rojas $L$, : Fil control de la tuberculosis. Rev Med Chile 1982; 110: 605-612.
9. Hamill P.V., Drizd T.A., Johnson C.L., Reed R.B., Roche A.F., Moore W.M.: Physical Growth: National Center for Health Statistics an percentiles. An J Clin Nutr 1979: 32; 607-629.

10. Slutkin G., Pérez-Stable E., Hopewell P.C.: Time course and Boosting of Tuberculin Reactions in Nursing Home Residents. Am Rey Respir Dis 1986: 134: 1048-1051.

11. Pérez-Siable E., Levin R., Pineda A., Sutkin G.: Tuberculin Skin Test Reactivity and Conversions in Lnited States and Foreign Born Latin Children. Pediati Infect Dis 1985; 4: 476-479.

12. Leaverton P.E.: A review of Biostatistics. Little Brown and Company, Boston, USA. 1978: 35-63.

13. Metha J.B.. Sukhani B., Saxena S.: BCG Vaccination in Newborn and Tuberculin Conversion. Indian Pediatr 1972; 9: 485-489.

14. Azzini 1 , Corrasco $R$., De la fuente $M$. Ferrer $X_{\text {. }}$ Latrach $C$., Ritus $P .:$ Estudio de campo de una vacuna BCG liofilizada. Rev Chil Pediatr 1985; 56: 234-237.

15. Azzini I, Carrasco R., De la Fuente M., Latrach C., Ferrer $X$., Rius $M$ : Reacción tuberculina $y$ cicatriz en lactantes vacunados con BCG ljofilizada. Rev Chil Pediatr 1984; 55: 313-316.

16. Grinduis H. Baynham M., Scott P., Thompson A., Wharton B.: Tuberculin Response two years after B.C.G. vaccination at birth. Arch Dis Child 1984:59: 614-619.

17. Magmus $K$. Edwards $L$.: The effect of repeated tuberculin testing on post vaccination alletgy. Lancet 2: 643-644, 1955.

18. Sepüleda R, Burr (r, Ferrer M., Sorensen $R$. Booster effect of tuberculin testing in healthy 16 yeat-old school children vaccinated with Bacillus Calmette-Guerin at Birth in Santiago, Chile. Pediatr Infect Dis $1988 ; 7$ : $578-581$. 